\title{
Frequency Selective Signal Extrapolation for Compensation of Missing Data in Sinograms
}

\author{
Joaquín López Herraiz, Student Member, IEEE, Samuel España, Student Member, IEEE, \\ Esther Vicente, Student Member, IEEE, Elena Herranz, Student Member, IEEE, \\ Manuel Desco, Juan José Vaquero, Senior Member, IEEE, José Udias, Member, IEEE
}

\begin{abstract}
We present a method to compensate for missing projection data in positron emission tomography (PET), which may result from gaps between adjacent detectors or from malfunctioning detectors. To prevent artifacts in the reconstruction when using Fourier rebinning (FORE) or analytical reconstruction algorithms, filling the data gaps is required. This new approach for sinogram data interpolation prior to reconstruction is based on a simple iterative frequency selective signal extrapolation method initially proposed and successfully applied for error concealment in image and video communication. In this work the method has been improved taking advantage of well known restrictions in the allowed region of frequencies of the sinograms.

We compare quantitatively the results of this technique with previously proposed gap filling procedures in both the sinograms and in their reconstructed images. The proposed technique outperforms those methods without requiring too much computational time.
\end{abstract}

\section{INTRODUCTION}

T PET scanners the acquired data are usually sampled at all

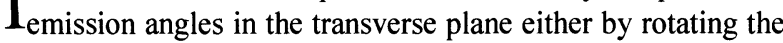
detectors around the source of activity or by using a complete ring of detectors. If the sampling is not uniform and complete, the task of reconstruction becomes more difficult. This can due to the geometrical design of the detector system or some malfunctioning detectors.

Because fully 3-D iterative reconstructions are computationally demanding, Fourier rebinning and 2-D reconstruction is sometimes preferred [1]. FORE requires a complete data set. Therefore, a reliable estimate of missing data is necessary [2]. Furthermore, fast analytic reconstruction techniques like Filtered Back-Projection (FBP) also require a complete sinogram as the missing data cause large artifacts in the reconstructed images.

Several methods to fill the data gaps in the sinograms have been previously proposed: a) linear interpolation in angles or

Manuscript received November 14, 2008. This work was supported in part by the MEC (FPA2007-07393), CDTEAM (CENIT-Ingenio 2010) Ministerio de Industria, Spain, CPAN (Consolider-Ingenio 2010) CSPD-2007-00042 projects, and the RECAVA-RETIC network. E. Vicente acknowledges support from a CSIC-JAE predoctoral fellowship.

J. L. Herraiz, S. España, E. Vicente, E. Herranz and J. M. Udias are with the Grupo de Física Nuclear, Dpto. Física Atómica, Molecular y Nuclear Universidad Complutense de Madrid, Spain (telephone: +34-91-394-4484, email: joaquin@nuclear.fis.ucm.es).

M. Desco and J. J. Vaquero are with the Unidad de Medicina y Cirugía Experimental, Hospital General Universitario Gregorio Marañón, Madrid, Spain (J.J. Vaquero e-mail: juanjo@hggm.es) bilinear interpolation in the radial and angular directions [3-5]; b) iterative constrained Fourier space method [6]; c) reconstruction of an intermediate volume [2-D ordered subsets expectation maximization (2-D OSEM)] based on direct planes or Fourier-rebinned sinograms, after which the image is forward projected to fill the gaps [5]; d) adaptive variational sinogram interpolation [7].

In this work we applied the method proposed in [8] for error concealment in image and video communication. The main idea is to describe the acquired sinogram as a weighted linear combination of periodic basis functions. The coefficients are obtained from all the available data outside the gaps. In general, periodic functions have good extrapolating properties as they are able to extend the signal periodically. In this work we will focus on the Discrete Fourier Transform (DFT) although other periodic functions like the Discrete Cosine Transform (DCT) or the Discrete Hilbert Transform (DHT) can be used.

Several iterative approaches are known for Fourier based signal extrapolation. The method of Karp et al. [6] uses the limited band-width of the sinogram spectrum (with bow-tie shape as described in [10]). This method follows the same scheme as previous proposed methods in image restoration [9] but taking advantage of the properties of the sinogram data. The spectrum of the observed signal, corrupted by the missing data, is first limited to the known allowed frequency region [10] and then transformed back to the projection space. After replacing the altered samples within the gaps, the sinogram is transformed again into the frequency domain, where the bowtie limitation is enforced. The extrapolation is obtained by iterating this procedure.

However, this method has some limitations. For example it cannot cope with vertical gaps in the sinogram (missing data along angles for a fixed radial bin). The constraint imposed in the Fourier space comes from the fact that no real sinusoidal trajectories in the sinogram can have a greater radius than the field-of-view (FOV) radius. Therefore if the gap radius [6] is lower or equal to the radius of the FOV, its trajectory in the projection data will be considered allowed and the method will not show a good performance. This happens for example in the MDAPET scanner [3]. This dependence of the performance of the constrained Fourier space method with the FOV of the scanner and the size of the object has not been clearly shown in the literature. 


\section{METHODS}

For a full description of the method applied, the reader is referred to [8]. For the sake of completeness a brief introduction is presented here.

We consider the sinogram S shown in Fig. 1. Our aim is to estimate the missing signal samples in the area $M$ from the acquired samples in the region $\mathrm{D}$. The extrapolation principle works as follows: the signal samples in the support area D are approximated by a weighted linear combination of basis functions which are defined over the entire sinogram $\mathrm{S}$. Therefore, each approximation over the support area D at the same time provides an estimation of the missing samples in $\mathrm{M}$.

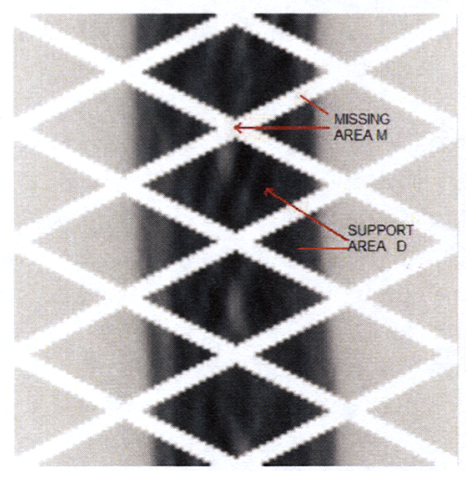

Fig. 1. Sinogram $\mathrm{S}$ with the missing area $\mathrm{M}$ and the acquired data $\mathrm{D}$

The values of the acquired data in the support area D are denoted by $\mathrm{f}[\mathrm{m}, \mathrm{n}]$, where $\mathrm{m}$ indicates the radial bin and $\mathrm{n}$ the angular bin. Our parametric model $g[\mathrm{~m}, \mathrm{n}]$ shall approximate the signal within the support area by a linear combination of the two-dimensional DFT basis functions:

$$
g[m, n]=\sum_{(k, l)} c[k, l] \cdot \varphi_{k, l}[m, n]
$$

Let us assume that at any step $v$ the parametric model approximating the signal in the support area is given by:

$$
g^{(\nu)}[m, n]=\sum_{(k, l)} c^{(\nu)}[k, l] \cdot \varphi_{k, l}[m, n]
$$

The residual error signal in the support area $(m, n) \in D$ at any iteration $v$ will be:

$$
r^{(\nu)}[m, n]=\left(f[m, n]-g^{(\nu)}[m, n]\right)
$$

Initially we start with a uniform zero parametric model:

$$
\begin{array}{ll}
g^{(\nu=0)}[m, n] & =0 \quad, \forall[\mathrm{m}, \mathrm{n}] \in \mathrm{S} \\
r^{(\nu=0)}[m, n] & =f[m, n], \forall[\mathrm{m}, \mathrm{n}] \in \mathrm{S}
\end{array}
$$

This iterative procedure approximates the signal within the support area updating the coefficients $\mathrm{c}^{(\mathrm{v})}[\mathrm{k}, \mathrm{l}]$ successively subject to an error energy constraint given by:

$$
E=\sum_{(m, n) \in D}\left(f[m, n]-g^{\left({ }^{\prime}\right)}[m, n]\right)^{2}
$$

The iteration stops when the residual error energy drops below a pre-specified threshold. The parametric model is then given in the entire area and the missing samples are obtained by an inherent extrapolation.
The steps followed in this restoration algorithm are:

1) Create a binary window of the support area of the sinogram:

$$
\omega[m, n]= \begin{cases}1, & (m, n) \in D \\ 0, & (m, n) \in M\end{cases}
$$

2) Perform the 2-D DFT of the mask and the initial residual:

$$
\begin{aligned}
W^{(0)}[k, l] & =\operatorname{DFT}\left\{\omega^{(0)}[m, n]\right\} \\
R^{(0)}[k, l] & =\operatorname{DFT}\left\{r^{(0)}[m, n]\right\}
\end{aligned}
$$

3) Search for the frequency $[u, v]$ with the largest value of the square of the residual error:

$$
[u, v]=\arg \max _{[k, l]}\left(R^{\left({ }^{\prime \prime}\right)}[k, l]\right)^{2}
$$

4) Obtain the correction to the coefficient c[u,v]:

$$
\Delta c[u, v]=R^{\left(\nu^{\prime \prime}\right.}[u, v]
$$

5) Update the coefficient $\mathrm{c}[\mathrm{u}, \mathrm{v}]$ and the residual error:

$$
\begin{aligned}
& c^{(\nu+1)}[u, v]=c^{(\nu)}[u, v]+\Delta c[u, v] \\
& R^{(\nu+1)}[k, l]=R^{(\nu)}[k, l] \\
& \quad-\Delta c[u, v] \cdot W[k-u, l-v] / W[0,0] \quad \forall k, l
\end{aligned}
$$

6) Repeat steps 3-5 until convergence is reached.

In this work the method has been improved taking advantage of well known restrictions in the allowed region of frequencies of the sinograms [10]. Updating only the allowed frequencies in the iterative procedure, a significant reduction in computational time is achieved.

The method proposed has been applied to a simulated acquisition obtained with PeneloPET [11]. The scanner simulated is composed of one ring of 8 rotating block detectors as shown in Fig. 2. One of the direct sinograms (without rebinning) was used as a test for the different methods and its FBP reconstruction was performed. After the simulation, gaps similar to those corresponding to the nonrotating scanner were added to the sinogram.

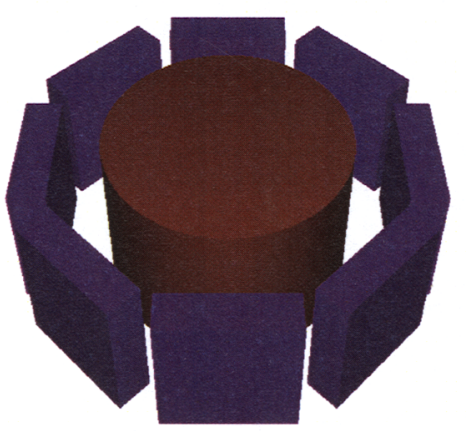

Fig. 2 - Simulated scanner with gaps between detectors

\section{RESULTS}

Fig. 3 shows the original sinogram with gaps and their corresponding restored sinograms after each method was 
applied. Fig. 4 shows the FBP reconstruction images (with Ramlak filter) from these sinograms and Fig. 5 shows profiles through these images.

The quantitatively evaluation of the method can be found in Table I. We define the error between the restored sinograms $\mathrm{f}$ respect to the gap-free sinogram $\mathrm{f}_{0}$ as:

$$
\operatorname{Error}_{S I N}(\%)=100 \cdot \sqrt{\frac{1}{N_{M}} \cdot \sum_{i \in M}\left(\frac{f[i]-f_{0}[i]}{f_{0}[i]}\right)^{2}}
$$

The index $\mathrm{i}$ runs over all $\mathrm{N}_{M}$ bins which belongs to the missing region $\mathrm{M}$.

We also define the error between the images $\mathrm{X}$ reconstructed from the restored sinograms and the image $\mathrm{X}_{0}$ obtained from the gap-free sinogram as:

$$
\operatorname{Error}_{I N G G}(\%)=100 \cdot \sqrt{\frac{1}{N_{V}} \cdot \sum_{i=1}^{N_{V}}\left(\frac{X[i]-X_{0}[i]}{X_{0}[i]}\right)^{2}}
$$

In this case we sum all the voxels $\mathrm{N}_{\mathrm{V}}$ of the image.

$$
\text { (c) }
$$

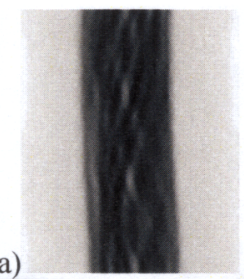

\section{(b)}

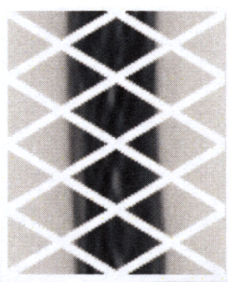

(e)

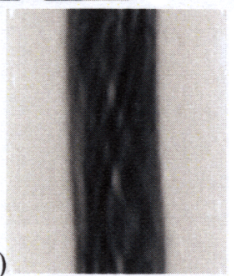

Fig 3 - 2D direct sinograms corresponding to a simulation of an Ultra micro resolution Phantom. Without gaps (a), with gaps and no gap-filling (b), with bilinear extrapolation fill (c), with Constrained Fourier method (d) and with the proposed Frequency Selective Signal Extrapolation method (e)

(a)

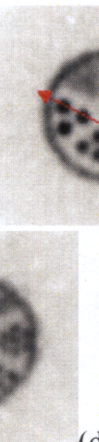

(b)

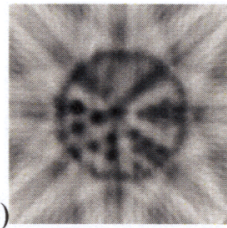

(c)

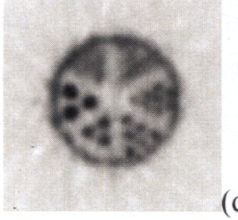

(d)

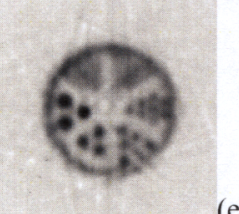

(e)

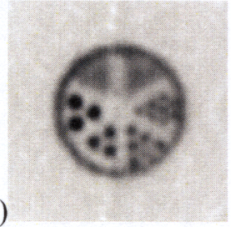

Fig. 4 - FBP reconstruction images from the sinograms of fig. 3. Without gaps (a), with gaps and no gap-filling (b), with Bilinear Extrapolation fill (c), with Constrained Fourier method (d) and with the proposed Frequency Selective Signal Extrapolation (e).

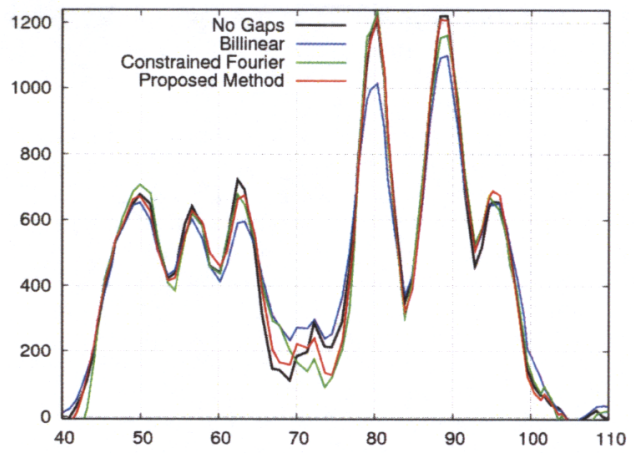

Fig. 5 -Profiles through the reconstructed images

TABLE I

QUANTIT ATIVE EVALUATION OF THE METHODS IN SINOGRAMS AND RECONSTRUCTED IMAGES

\begin{tabular}{|c|c|c|c|c|}
\hline Error (\%) & $\begin{array}{c}\text { Original } \\
\text { with } \\
\text { gaps }\end{array}$ & Bilinear & $\begin{array}{c}\text { Constrained } \\
\text { Fourier }\end{array}$ & $\begin{array}{c}\text { Frequency } \\
\text { Selective } \\
\text { Extrapol. }\end{array}$ \\
\hline Sinograms & $100 \%$ & $9 \%$ & $11 \%$ & $7 \%$ \\
\hline Images & $87 \%$ & $24 \%$ & $23 \%$ & $14 \%$ \\
\hline
\end{tabular}

\section{CONCLUSIONS}

A new method is presented which compensates for missing projection data in sinograms. This method allows the use of filtered back-projection reconstruction for 2-D sinograms or FORE for 3-D acquired data. The proposed technique is fast and the reconstructed images show significant improvements respect to previous proposed methods. It uses only the acquired data outside the gaps to obtain the coefficients of the Fourier Transform of the whole projection data. The convergence of the method and its independence on the geometry of the scanner and the gaps makes it robust and reliable.

\section{ACKNOWLEDGMENT}

Part of the computations of this work were done at the "High Capacity Cluster for Physical Techniques" of the Faculty for Physical Sciences of the UCM, funded in part by the UE under the FEDER program and in part by the UCM.

\section{REFERENCES}

[1] C. Michel, M. Schmand, X. Liu, M. Sibomana, S. Vollmar, C. Knoss, M. Lercher, C. Watson, D. Newport, M. Casey, M. Defrise, K. Wienhard and W. D. Heiss, "Reconstruction strategies for the HRRT," IEEE Nucl. Sci. Symp. and Med. Imag. Conf., 2000.

[2] M. Defrise, P. E. Kinahan, D. W. Townsend, C. Michel, M. Sibomana and D. F. Newport, "Exact and approximate rebinning algorithms for 3-D PET dat,". IEEE Trans. Med. Imag., vol. 16, no. 2, pp. 1+5-158, 1997.

[3] H. Baghaei, H. Li, J. Uribe, Y. Wang and W. H. Wong, "Compensation of missing projection data for MDAPET camera," IEEE Nucl. Sci. Symp. and Med. Imag. Conf., 2000.

[4] R. Buchert, K. H. Bohuslavizki, J. Mester and M. Clausen, "Quality Assurance in PET: Evaluation of the Clinical Relevance of Detector Defects, "J. Nucl. Med., vol. 40, no. 10, pp. 1657-1665, 1999. 
[5] H. W. A. M. de Jong, R. Boellaard, C. Knoess, M. Lenox, C. Michel, M. Casey and A. A. Lammertsma, "Correction methods for missing data in sinograms of the HRRT PET scanner," IEEE Trans. Med. Imag. vol. 50, no. 5, pp. 1+52-1+56, Oct. 2003.

[6] J. S. Karp, G. Muehllehner and R.M. Lewitt, "Constrained Fourier space method for compensation of missing data in emission computed tomography," IEEE Trans. Med. Imag. vol. 7, no. 1, pp. 21-25, 1988.

[7] H. Kostler, M. Prummer, U. Rude and J. Hornegger, "Adaptive variational sinogram interpolation of sparsely sampled CT data," in 18th International Conference on Pattern Recognition, 2006.

[8] A. Kaup, K. Meisinger, and T. Aach, "Frequency selective signal extrapolation with applications to error concealment in image communication," Int. J. of Electronics and Communications, vol. 59 , no. 3, pp. 147-156, 2005.

[9] A. Papoulis, "A new algorithm in spectral analysis and band-limited extrapolation," IEEE Trans. on Circuits and Systems, vol. 22, no. 9, pp. $735-7+2,1975$.

[10] Rattey, P. and A. Lindgren, "Sampling the 2-D Radon transform," IEEE Trans. on Acoustics, Speech, and Signal Processing, vol. 29, no. 5, pp. 99+-1002, 1981.

[11] S. Espana, J. L. Herraiz, E. Vicente, E. Herranz, J. J. Vaquero, M. Desco, J. M. Udias, "PeneloPET, a Monte Carlo PET simulation toolkit based on PENELOPE: Features and Validation," in IEEE Nucl. Sci. Symp. and Med. Imag. Conf., 2006. 